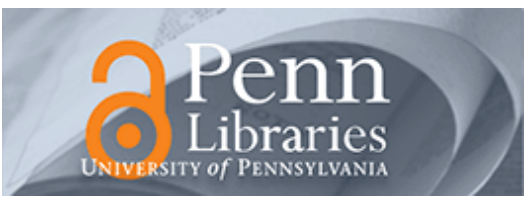

University of Pennsylvania

ScholarlyCommons

$4-13-2010$

\title{
Helical Packings and Phase Transformations of Soft Spheres in Cylinders
}

\author{
Matthew A. Lohr \\ University of Pennsylvania, mlohr@sas.upenn.edu
}

A. M. Alsayed

University of Pennsylvania

Zheng Zhang

University of Pennsylvania

Bryan G. Chen

University of Pennsylvania, chb@sas.upenn.edu

Randall D. Kamien

University of Pennsylvania, kamien@physics.upenn.edu

Follow this and additional works at: https://repository.upenn.edu/physics_papers

a next page for additional authors

Part of the Physics Commons

\section{Recommended Citation}

Lohr, M. A., Alsayed, A. M., Zhang, Z., Chen, B. G., Kamien, R. D., \& Yodh, A. G. (2010). Helical Packings and Phase Transformations of Soft Spheres in Cylinders. Retrieved from https://repository.upenn.edu/

physics_papers/16

\author{
Suggested Citation: \\ Lohr, M.A., A.M. Alsayed, B.G. Chen, Z. Zhang, R.D. Kamien and A.G. Yodh. (2010). "Helical packings and phase \\ transformations of soft spheres in cylinders." Physical Review E 81, 040401(R). \\ (C) The American Physical Society \\ http://dx.doi.org/10.1103/PhysRevE.81.040401 \\ This paper is posted at ScholarlyCommons. https://repository.upenn.edu/physics_papers/16 \\ For more information, please contact repository@pobox.upenn.edu.
}




\title{
Helical Packings and Phase Transformations of Soft Spheres in Cylinders
}

\author{
Abstract \\ The phase behavior of helical packings of thermoresponsive microspheres inside glass capillaries is \\ studied as a function of the volume fraction. Stable packings with long-range orientational order appear to \\ evolve abruptly to disordered states as the particle volume fraction is reduced, consistent with recent \\ hard-sphere simulations. We quantify this transition using correlations and susceptibilities of the \\ orientational order parameter 6 . The emergence of coexisting metastable packings, as well as coexisting \\ ordered and disordered states, is also observed. These findings support the notion of phase-transition-like \\ behavior in quasi-one-dimensional systems. \\ Disciplines \\ Physical Sciences and Mathematics | Physics \\ Comments \\ Suggested Citation: \\ Lohr, M.A., A.M. Alsayed, B.G. Chen, Z. Zhang, R.D. Kamien and A.G. Yodh. (2010). "Helical packings and \\ phase transformations of soft spheres in cylinders." Physical Review E 81, 040401(R). \\ (c) The American Physical Society \\ http://dx.doi.org/10.1103/PhysRevE.81.040401 \\ Author(s) \\ Matthew A. Lohr, A. M. Alsayed, Zheng Zhang, Bryan G. Chen, Randall D. Kamien, and Arjun G. Yodh
}




\title{
Helical packings and phase transformations of soft spheres in cylinders
}

\author{
M. A. Lohr, ${ }^{1}$ A. M. Alsayed, ${ }^{1,2}$ B. G. Chen, ${ }^{1}$ Z. Zhang, ${ }^{1,2}$ R. D. Kamien, ${ }^{1,3}$ and A. G. Yodh $^{1}$ \\ ${ }^{1}$ Department of Physics and Astronomy, University of Pennsylvania, Philadelphia, Pennsylvania 19104, USA \\ ${ }^{2}$ Complex Assemblies of Soft Matter, CNRS-Rhodia-UPenn UMI 3254, Bristol, Pennsylvania 19007, USA \\ ${ }^{3}$ School of Mathematics, Institute for Advanced Study, Princeton, New Jersey 08540, USA
}

(Received 17 December 2009; published 13 April 2010)

\begin{abstract}
The phase behavior of helical packings of thermoresponsive microspheres inside glass capillaries is studied as a function of the volume fraction. Stable packings with long-range orientational order appear to evolve abruptly to disordered states as the particle volume fraction is reduced, consistent with recent hard-sphere simulations. We quantify this transition using correlations and susceptibilities of the orientational order parameter $\psi_{6}$. The emergence of coexisting metastable packings, as well as coexisting ordered and disordered states, is also observed. These findings support the notion of phase-transition-like behavior in quasi-one-dimensional systems.
\end{abstract}

DOI: 10.1103/PhysRevE.81.040401

PACS number(s): 64.70.dj, 64.70.dm, 64.75.Xc, 64.75.Yz

The phenomenology of ordered phases and phase transformations in systems with low dimensionality is surprisingly rich. While dense three-dimensional (3D) thermal packings can exhibit long-range order, for example, this trait is absent in purely one-dimensional (1D) systems [1]. Complexities arise, however, when considering 3D systems confined primarily to one dimension. Investigation of order and phase behavior in quasi-1D thermal systems, therefore, holds potential to elucidate a variety of novel physical processes that have analogies with polymer folding [2], formation of supermolecular fibers in gels [3], and emergence of helical nanofilaments in achiral bent-core liquid crystals [4].

Packings of soft colloidal spheres in cylinders provide a useful model experimental system to quantitatively investigate order and phase transformations in quasi-onedimension. At high densities, spheres in cylindrical confinement are predicted to form helical crystalline structures $[5,6]$. Evidence for such packings has been found in foams $[7,8]$, biological microstructures [5], colloids in microchannels [9], and fullerenes in nanotubes [10]. However, research on these systems has been limited to static snapshots and athermal media. Recent simulations suggest that transitions between different helical ordered states $[11,12]$ and between quasi-1D ordered and disordered states [13-15] should exist in thermal systems, but such transitions have not been investigated experimentally.

In this Rapid Communication, we explore ordered and disordered structures in a quasi-1D thermal system of soft particles with adjustable volume fraction. In particular, we create helical packings of thermoresponsive colloid particles in glass microcapillaries, we show theoretically that phases with long-range orientational order can exist in quasi-onedimension, we demonstrate experimentally that such phases with long-range orientational order exist at volume fractions below maximal packing, and we study volume-fractioninduced melting of these orientationally ordered phases into liquid phases. The orientational order parameters and susceptibilities that characterize these phases and this crossover are measured and analyzed. Coexisting regions of ordered and disordered states and coexisting ordered domains with different pitches and chiralities are observed at these crossover points. Such coexistence effects suggest the presence of abrupt or discontinuous volume-fraction-driven transitions in quasi-1D structures. Interestingly, these orientationally ordered phases in quasi-one-dimension share physical features with orientationally ordered phases observed $[16,17]$ and predicted [18] in two dimensions.

The experiments employed aqueous suspensions of rhodamine-labeled poly- $N$-isopropylacrylamide (NIPA) microgel spheres (polydispersity $\leq 3 \%$ ) with diameters which decrease linearly and reversibly with increasing temperature [19]. The unique thermoresponsive characteristics of NIPA microgel particles provide a means to explore the phase behavior $[17,20,21]$ of soft spheres in quasi-one-dimension as a function of the volume fraction. Borosilicate glass tubes (McMaster Carr) were heated and stretched to form microcapillaries with inner diameters comparable to the NIPA microsphere diameter. An aqueous suspension of NIPA microspheres was then drawn into the capillaries; subsequently, the capillary ends were sealed with epoxy and attached to a glass microscope slide. Samples were annealed at $28{ }^{\circ} \mathrm{C}$ to permit uneven packings to rearrange at low volume fraction, thereby creating stable high-volume-fraction packings when returned to lower temperatures.

The samples were imaged under a $100 \times$ oil-immersion objective (numerical aperture $=1.4$ ) using spinning-disk confocal microscopy (QLC-100, Visitech, International). Resulting images depict $75-\mu \mathrm{m}$-long segments of densely packed regions which span at least several hundred microns. An objective heater attached to the microscope (Bioptechs) permitted the control of the sample temperature to within $\pm 0.1{ }^{\circ} \mathrm{C}$. Standard particle tracking routines [22] were employed to identify particle positions from three-dimensional image stacks and two-dimensional cross sections.

At high densities, we observe crystalline helical packings with varying pitch and chirality dependent on the particle and tube diameters. The observation of large ordered domains is consistent with the tendency for these nearly monodisperse particles to form uniform crystals in two [17] and three [20] dimensions. Particles in hard-sphere helical packings predicted for cylinders [5] have six nearest neighbors whose relative order along the tube axis corresponds to a characteristic set of three integers $(m, n, m+n)$. This notation for distinct helical crystalline structures is commonly used in 


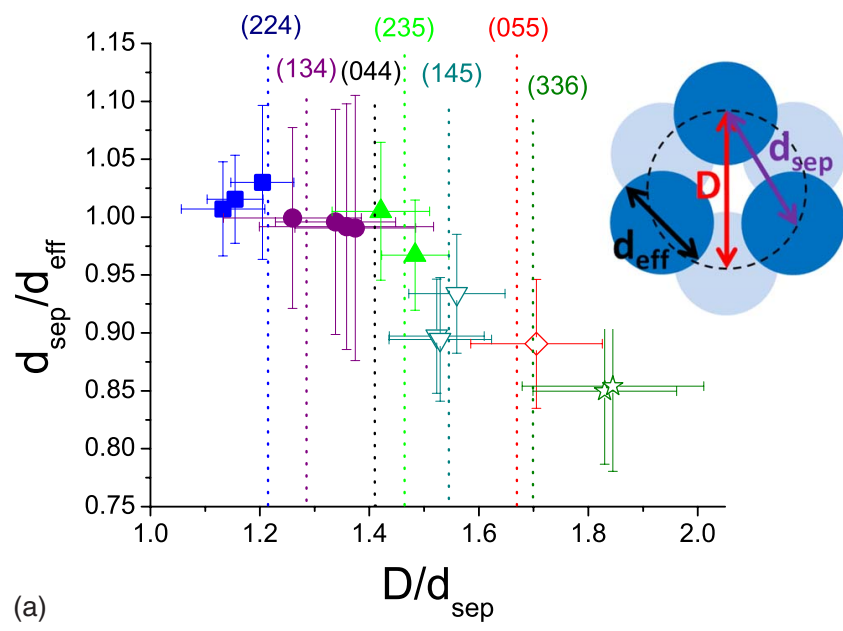

(b)

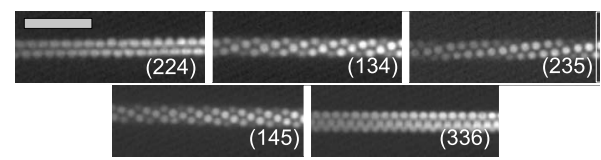

FIG. 1. (Color online) (a) Geometrical characteristics of observed structures: $(2,2,4)$ (blue $\square$ ), $(1,3,4)$ (purple $\bigcirc$ ), $(2,3,5$ ) (green $\triangle$ ), $(1,4,5)($ cyan $\nabla),(0,5,5)$ (red $\diamond)$, and $(3,3,6)$ (dark green $\star)$. Filled symbols indicate structures with observable Brownian motion. Dashed vertical lines indicate theoretical $D / d_{\text {sep }}$ values for predicted structures. Inset: cartoon of axial cross section of a (336) structure with $d_{\text {sep }}>d_{\text {eff. }}$. (b) Confocal fluorescence images of helical NIPA packings at high volume fraction. Scale bar $=10 \mu \mathrm{m}$.

phyllotaxy and is similar to the vector used to describe carbon nanotube chirality. We verify such crystalline ordering from the analysis of 3D confocal image stacks. All varieties of predicted helical packings in the given range of tubediameter-to-particle-diameter ratios were observed in 15 samples (Fig. 1), with the exception of structure $(0,4,4)$. Additionally, the ratio $D / d_{\text {sep }}$, where $D$ is twice the average radial distance from the particle centers to the central axis of the tube and $d_{\text {sep }}$ is the average nearest-neighbor particle separation, fell within experimental error of the predicted maximally packed hard-sphere values.

Ordered structures were found to exist over a range of

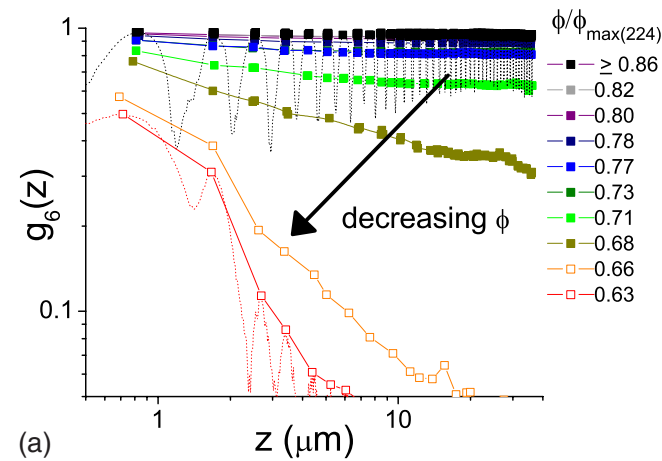

volume fractions below maximal packing. When the effective particle diameter $d_{\text {eff }}$ [23] was such that $d_{\text {sep }} / d_{\text {eff }}$ $<0.95$, the particles did not appear to move (i.e., motions greater than $0.2 \mu \mathrm{m}$ were not observed during the $10 \mathrm{~s}$ scan). In such cases, we consider the particles to be packed closer than their effective diameters. For $d_{\text {sep }} / d_{\text {eff }}>0.95$, particles fluctuate significantly about their equilibrium positions; thus, thermal helical crystalline structures exist at volume fractions below close packing.

The volume fractions of such samples were then lowered further to determine if, when, and how the packings disorder to isotropic states. Two uniformly packed samples were chosen and are presented here for careful analysis: a $(2,2,4)$ packing of microspheres with a diameter of $1.71 \mu \mathrm{m}$ at $22{ }^{\circ} \mathrm{C}$ and a $(0,6,6)$ packing of microspheres with a diameter of $1.23 \mu \mathrm{m}$ at $22{ }^{\circ} \mathrm{C}$. The sample temperature was increased in steps of $0.2-0.7^{\circ} \mathrm{C}$. At each temperature step, after allotting an ample time for the sample to reach thermal equilibrium (at least $5 \mathrm{~min}$ ), videos of two-dimensional cross sections of the packings were taken at 15-30 frames per second for approximately $5 \mathrm{~min}$. Although these two-dimensional videos lose some of the structural information available in three-dimensional scans, they provide data at much higher speeds and yield better axial position tracking of particles in view.

A local orientational order parameter, $\psi_{6 j}=\sum_{k}^{N_{n n}} e^{6 i \theta_{j k} / N_{n n}}$, quantifies helical order in these systems. Here, $\theta_{j k}$ is the angle between the axis of the tube and the bond between particles $j$ and $k$, and $N_{n n}$ is the number of nearest neighbors of particle $j$. Although this order parameter is typically used for two-dimensional planar systems, it is acceptable to use in the analysis of two-dimensional slices of helical packings. Helical packings are effectively two-dimensional triangular lattices wrapped into cylinders, and the observed cross sections of these particular packings exhibit only a slight variation from the ideal two-dimensional hexagonal lattice.

We examined the spatial extent of orientational order along the tube by calculating the orientational spatial correlation function $g_{6}\left(z=\left|z_{j}-z_{k}\right|\right)=\left\langle\psi_{6 j}^{*} \psi_{6 k}\right\rangle$, where $z_{j}$ is the axial position of particle $j$. As depicted in Fig. 2, the resulting correlation functions decrease quickly at low volume fractions, as expected in a disordered state. However, at high

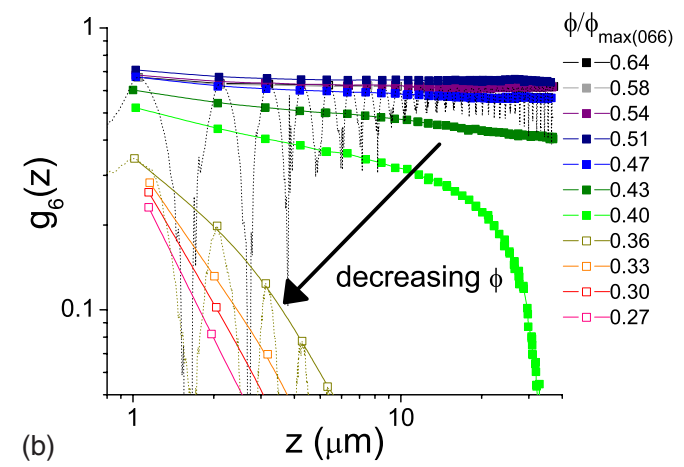

FIG. 2. (Color online) Local maxima of orientational spatial correlation function $g_{6}(z)$ for samples with $($ a) $(2,2,4)$ and $(b)(0,6,6)$ packings. Dashed lines: full correlation function at (a) $\phi / \phi_{\max (224)}=0.86$ and 0.63 , and (b) $\phi / \phi_{\max (066)}=0.64$ and 0.36 , where $\phi_{\max }$ is the maximum volume fraction for a given packing of hard spheres with the observed particle spacing. Empty symbols indicate samples with short-range order. Oscillations arise from the periodic distribution of particle positions along the tube axis. Full correlation functions were calculated for all volume fractions, but omitted for the sake of clarity. 
volume fractions, these functions exhibit long-range order within the experiment's field of view. These experimental observations are consistent with an expectation of long-range orientational order. Although long-range translational order is impossible in one dimension at finite temperature [1], long-range orientational order is possible, just as in the much-storied theory of two-dimensional melting [24].

One can show theoretically that long-range orientational order persists even in this quasi-one-dimensional system by evaluating the orientational correlation function $g_{6}(\mathrm{r})$ using the isotropic elasticity free energy [24,25] in an "unwrapping" of the particles on the cylinder surface onto a twodimensional infinitely long strip [23]. We assume that particles are packed densely enough, so that fluctuations along the radial direction of the cylinder may be neglected. As $r \rightarrow \infty, g_{6}$ approaches a constant. Thus, finite correlations exist at infinite distance, a hallmark of a phase with long-range order. Orientational order arises here through the crystalline axes defined by unwrapping the cylinder, rather than through an explicit additional mode, as in such systems studied in [26].

To our knowledge, the existence of long-range orientational order has not been characterized in previous studies of packing in cylindrical systems [11-15]. At low volume fractions, one expects long-range orientational correlations to disappear as observed in the experiment. However, suggestive as this combination of theory and experiment may be, we emphasize that further work is required to elucidate whether a generalization of the Kosterlitz-ThoulessHalperin-Nelson-Young (KTHNY) theory [24] is appropriate for this system.

To quantify the crossover from long-range to short-range order in the experimental system, an average orientational order parameter $\left|\psi_{6}\right|$ is defined for each frame, where $\psi_{6}$ $=\frac{1}{N} \sum_{j}^{N} \psi_{6 j}$. The height of the first peak of the one-dimensional axial structure factor $S\left(k_{\max }\right)$, where $S(k)=[N(N$ $-1)]^{-1}\left|\sum \sum e^{i k \mid z_{l}-z_{m}}\right| \mid$, is used as a translational order parameter. Here, $z$ denotes the axial position of each particle, $N$ is the number of particles in the field of view at a given time, and $k_{\text {max }}$ is chosen iteratively for each volume fraction.

In Figs. 3(a) and 3(b), it is evident that both the average translational and orientational order parameters cross from an ordered state at high volume fraction to a disordered state at low volume fraction, although the change in $\left|\psi_{6}\right|$ is significantly sharper than the change in $S\left(k_{\max }\right)$. The orientational crossover complements a recent simulation which finds a similar crossover in hard-sphere packings with decreasing density [14].

To characterize the fluctuations in orientational order, we calculate the orientational susceptibility $\chi_{6}=\left\langle\left|\psi_{6}\right|^{2}\right\rangle-\left\langle\left|\psi_{6}\right|\right\rangle^{2}$, where $\langle\cdot\rangle$ represents the time average. Statistical effects of the finite size of the system are accounted for by calculating the susceptibility of different subsegments of length $L$ in the system and extrapolating to the limit $L \rightarrow \infty$, similar to the calculations in [17]. Plots of $\chi_{6}$ in Figs. 3(c) and 3(d) clearly demonstrate a peak in the orientational susceptibility. The location of this peak coincides with the onset of both orientational and translational orders in the system. Translational susceptibilities were also calculated, but did not exhibit clear peaks or trends with respect to the order parameters or the
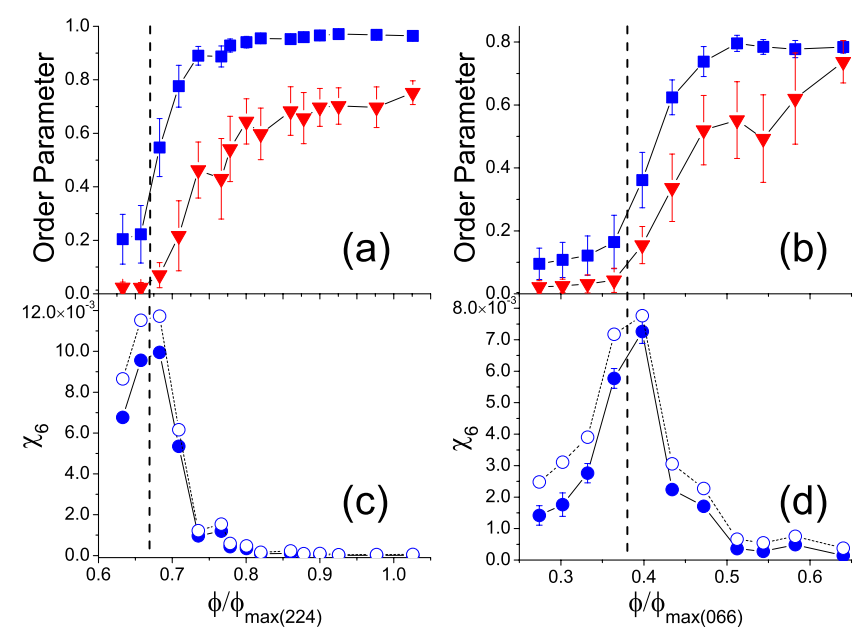

FIG. 3. (Color online) Top: mean values of translational (red $\boldsymbol{\nabla}$ ) and orientational (blue $\mathbf{\square}$ ) order parameters for (a) $(2,2,4)$ and (b) $(0,6,6)$ samples. Bottom: orientational susceptibilities for (c) $(2,2,4)$ and (d) $(0,6,6)$ systems, for sample size $=75 \mu \mathrm{m}(\bigcirc)$ and $\infty(\bullet)$. Dashed vertical lines at $\phi / \phi_{\max (224)}=0.67$ and $\phi / \phi_{\max (066)}=0.38$.

volume fraction. We do not expect any transitionlike behavior from translational susceptibilities due to arguments from [1] against long-range translational order, which apply in quasi-one-dimension.

The existence of a diverging peak in the susceptibility of an order parameter typically indicates a phase transition [27]. However, it is difficult from the given data points to determine if this is truly a diverging peak, and whether it would indicate a first-order (asymmetrically diverging) or secondorder (symmetrically diverging) phase transition. Upon closer examination of the $(0,6,6)$ sample, we observe the coexistence of ordered and disordered domains for $\phi / \phi_{\max (066)}=0.47-0.40$ [see Fig. $4(\mathrm{a})$ ]. We also observe the appearance of a small domain with dubious order in the $(2,2,4)$ sample at $\phi / \phi_{\max (224)}=0.68$. The appearance of these coexisting domains is consistent with the spatial correlation functions exhibiting neither long-range nor short-range be-

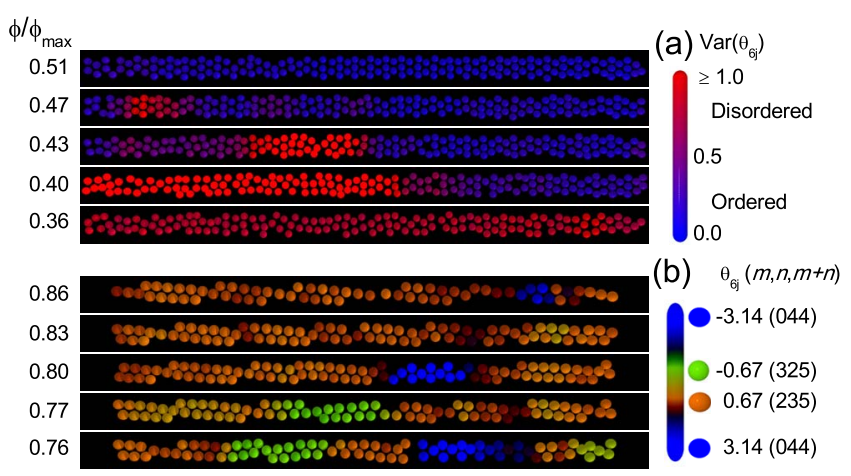

FIG. 4. (Color online) Snapshots of particle tracks from systems exhibiting emergence of coexisting domains. (a) $(0,6,6)$ structure with emerging disordered domains. Color represents local variance of the phase of $\psi_{6},\left\langle\theta_{6 j}^{2}\right\rangle-\left\langle\theta_{6 j}\right\rangle^{2}$, in $1.2 \mu \mathrm{m}$ segments. (b) $(2,3,5)$ structure with emerging domains of $(3,2,5)$ and $(0,4,4)$ structures. Color represents phase of $\psi_{6 j}, \theta_{6 j}$, which characterizes packing orientation. Structures corresponding to each $\theta_{6 j}$ are given on the right. 
havior at intermediate volume fractions in Fig. 2 $\left(\phi / \phi_{\max (066)}=0.40, \phi / \phi_{\max (224)}=0.68\right)$. The presence of such a solid-liquid coexistence has also been seen in recent simulations of hard spheres in cylinders [15].

In other samples, domains with different helical orders often appear as the volume fraction decreased [Fig. 4(b)]. The appearance of these domains was difficult to quantify since domains would grow, shrink, and/or disappear with decreasing volume fraction. The structures observed in coexisting states were those with the most similar predicted linear densities and $D / d_{\text {sep }}$ values, consistent with recent hardsphere simulations [11,12]. This coexistence of ordered structures should not be confused with dislocation-mediated structural transitions in athermal helical crystals theoretically studied in [6] and observed in [8], especially because of the difference in the observed sequence of coexisting structures.
In summary, we created ordered helical packings of thermoresponsive colloids and observed the presence of longrange order resilient to thermal fluctuations. Sharp crossovers from orientationally ordered to disordered phases with decreasing volume fraction were observed. In addition, we find basic evidence for abrupt volume-fraction-driven structure-to-structure transitions. These findings raise and elucidate fundamental questions on the subject of melting in one dimension.

We especially thank Yilong Han for clarifying discussions about experimental analyses, and we also thank Tom Haxton, Yair Shokef, and Peter Yunker for enlightening discussions. This work was supported by MRSEC Grant No. DMR0520020, NSF Grant No. DMR-080488, and NASA Grant No. NNX08AO0G.
[1] L. Van Hove, Physica 16, 137 (1950).

[2] L. E. Hough et al., Science 325, 456 (2009).

[3] Y. Q. Zhou, C. K. Hall, and M. Karplus, Phys. Rev. Lett. 77, 2822 (1996).

[4] J. F. Douglas, Langmuir 25, 8386 (2009).

[5] R. O. Erickson, Science 181, 705 (1973).

[6] W. F. Harris and R. O. Erickson, J. Theor. Biol. 83, 215 (1980).

[7] N. Pittet, N. Rivier, and D. Weaire, Forma 10, 65 (1995).

[8] P. Boltenhagen and N. Pittet, EPL 41, 571 (1998); N. Pittet et al., ibid. 35, 547 (1996); P. Boltenhagen, N. Pittet, and N. Rivier, ibid. 43, 690 (1998).

[9] J. H. Moon et al., Langmuir 20, 2033 (2004); F. Li et al., J. Am. Chem. Soc. 127, 3268 (2005); M. Tymczenko et al., Adv. Mater. 20, 2315 (2008).

[10] A. N. Khlobystov, D. A. Britz, A. Ardavan, and G. A. Briggs, Phys. Rev. Lett. 92, 245507 (2004); T. Yamazaki et al., Nanotechnology 19, 045702 (2008).

[11] G. T. Pickett, M. Gross, and H. Okuyama, Phys. Rev. Lett. 85, 3652 (2000).

[12] K. Koga and H. Tanaka, J. Chem. Phys. 124, 131103 (2006).

[13] M. C. Gordillo, B. Martinez-Haya, and J. M. Romero-Enrique, J. Chem. Phys. 125, 144702 (2006).

[14] F. J. Duran-Olivencia and M. C. Gordillo, Phys. Rev. E 79, 061111 (2009).

[15] H. C. Huang, S. K. Kwak, and J. K. Singh, J. Chem. Phys. 130, 164511 (2009).
[16] C. A. Murray and D. H. VanWinkle, Phys. Rev. Lett. 58, 1200 (1987); K. Zahn, R. Lenke, and G. Maret, ibid. 82, 2721 (1999).

[17] Y. Han, N. Y. Ha, A. M. Alsayed, and A. G. Yodh, Phys. Rev. E 77, 041406 (2008).

[18] K. J. Strandburg, Rev. Mod. Phys. 60, 161 (1988).

[19] B. R. Saunders and B. Vincent, Adv. Colloid Interface Sci. 80, 1 (1999); R. Pelton, ibid. 85, 1 (2000); L. A. Lyon et al., J. Phys. Chem. B 108, 19099 (2004).

[20] A. M. Alsayed et al., Science 309, 1207 (2005).

[21] H. Senff and W. Richtering, J. Chem. Phys. 111, 1705 (1999); J. Wu, B. Zhou, and Z. Hu, Phys. Rev. Lett. 90, 048304 (2003); Y. Han et al., Nature (London) 456, 898 (2008); Z. Zhang et al., ibid. 459, 230 (2009); P. Yunker, Z. Zhang, K. B. Aptowicz, and A. G. Yodh, Phys. Rev. Lett. 103, 115701 (2009); J. Brijitta et al., J. Chem. Phys. 131, 074904 (2009).

[22] J. C. Crocker and D. G. Grier, J. Colloid Interface Sci. 179, 298 (1996).

[23] See supplementary material at http://link.aps.org/supplemental/ 10.1103/PhysRevE.81.040401 for details of particle diameter characterization and for additional details of this calculation.

[24] D. R. Nelson and B. I. Halperin, Phys. Rev. B 19, 2457 (1979).

[25] N. D. Mermin, Phys. Rev. 176, 250 (1968).

[26] Y. Kantor and M. Kardar, Phys. Rev. E 79, 041109 (2009).

[27] K. Binder, Rep. Prog. Phys. 50, 783 (1987). 